\title{
Evaluation of a PRImary-ORthopaedic (PRIOR) low back pain care model in Hong Kong: a pilot study
}

\section{Shirley Yue Kwan Choi}

Hospital Authority

\section{Sheung Wai Law}

Tai Po Hospital

\section{William WL Wong}

Prince of Wales Hospital

\section{Jamie Lau}

Prince of Wales Hospital

Shaau Yiu Ko

Tai Po Hospital

Sibei Lin

Chinese University of Hong Kong

\section{Eric Hui}

Hospital Authority

\section{Samuel Yeung Shan Wong}

The Chinese University of Hong Kong

\section{Regina Wing Shan Sit ( $\nabla$ reginasit@cuhk.edu.hk)}

Chinese University of Hong Kong https://orcid.org/0000-0002-4717-7773

\section{Research article}

Keywords: Primary-Orthopaedic low back pain care model, effectiveness, health service

Posted Date: January 8th, 2020

DOI: https://doi.org/10.21203/rs.2.20340/v1

License: (c) (1) This work is licensed under a Creative Commons Attribution 4.0 International License. Read Full License 


\section{Abstract}

Introduction: Low back pain (LBP) is a common and disabling condition with significant socioeconomic burden. A LBP care delivery model with collaborative input from primary care physicians, orthopaedic surgeons and allied health professionals has been implemented in one of the local tertiary hospital to streamline LBP care.

Objective: To evaluate the preliminary effectiveness of the primary-orthopaedic (PRIOR) LBP care model.

Methods: This was a 6-month quasi-experimental study with pre and post assessment. Participants $(\mathrm{N}=30)$ with chronic LBP were recruited and stratified by physiotherapists at the triage station located at Orthopaedic Special Outpatient Clinic. Eligible participants were referred to attend primary physicians with trained musculoskeletal skills for risk-matched treatment at Family Medicine Clinic. Patient-specific outcomes included the acceptance rate, Roland Morris Disability (RMD) scores, the self-pain efficacy scores (PSE) scores and the Euro-Qol-5D. The health service outcomes included the waiting time, discharge rate, adherence rate and healthcare resources utilization.

Results: Of the 33 participants screened, 30 were eligible and enrolled for the study, the recruitment rate was $91 \%(X 2=0.569, p=0.451)$. At 6 months, participants reported overall improvement of the RMD scores ( $\beta$ coefficient $-4.3,95 \%$ confidence interval $\mathrm{Cl}-5.9$ to $-2.7, \mathrm{P}<0.001$ ) and PSEQ scores ( $\beta$ coefficient $7.3,95 \% \mathrm{Cl}$ 3.0-12.0, $P=0.002)$. The waiting time of the FM clinic was $11.6 \pm 1.6$ weeks, the overall discharge rate was $73 \%$, and the adherence rate was $87 \%$.

Conclusions: The study suggested that the PRIOR LBP model could potentially improve the clinical outcomes of LBP patients and enhance the overall LBP health service delivery.

\section{Introduction}

Low back pain (LBP) is a highly prevalent, disabling and costly condition ${ }^{1,2}$ According to the Global Burden of Disease Study in 2017, around 570 million people were suffering from activity-limiting LBP and it is now the number one cause of disability globally. ${ }^{3}$ The burden is expected to grow with the increasing and ageing population. ${ }^{1,4}$ The direct and indirect costs of CLBP are substantial in all developed countries. ${ }^{5,6}$

LBP is a symptom rather than a disease. Rarely can a specific cause of low back pain be identified; thus, most LBP is termed non-specific, which accounted for more than $90 \%$ of the clinical presentation. ${ }^{7}$ Majority of the LBP patients can be managed with education on self-management, medications, physical therapy and timely review; only small proportion of them need specific surgical treatments. ${ }^{8}$ Although clinical guidelines suggest that most LBP should be managed in primary care, many patients are still referred to orthopaedic (ORT) specialist outpatient clinics (SOPC) for surgical review. ${ }^{9}$ Inappropriate or overuse of specialist referrals not only lead to long waiting time but also delay the care of appropriate candidates for surgical interventions. 
Locally, the ORT SOPCs received LBP referrals from the Accidental and Emergency Departments, the public and private out-patient clinics, and from all specialities. With the huge numbers of referrals every day, the average waiting time was long. ${ }^{10}$ Despite the growing evidence of various effective treatment, timely access remains a major obstacle for our LBP patients. ${ }^{11}$ Study has shown that patients with LBP with long-term pain and disability for more than a year are unlikely to return to their normal activities. ${ }^{12}$ In addition, those who have been off work with chronic LBP for 1 to 2 years are also unlikely to return to any form of work in the foreseeable future. ${ }^{13}$ Thus, the long waiting time may have a significant contribution to the development of the heavy disease burden. ${ }^{14}$

In view of this, a Primary-Orthopaedic (PRIOR) LBP care model based on the context of local healthcare system was developed in October 2017 by the Department of the Family Medicine and Primary Care and the Department of Orthopaedic and Traumatology, Prince of Wales Hospital (PWH), the Hong Kong Special Administrative Region. The care model used a triage approach at the ORT SOPC to stratify LBP patients with their prognostic risks and referred them to consult primary care physicians trained with musculoskeletal skills at Family Medicine-Orthopaedic Clinic (FM-ORT) for early assessment and managment. ${ }^{15}$

\section{Aims and hypothesis:}

The aims of this project were to evaluate the preliminary effectiveness of a pilot LBP care model by measuring patient-specific and health service outcomes.

\section{Methods}

\section{Study Design:}

The study was a 6-month quasi-experimental study with pre and post assessment.

\section{Participants and settings:}

LBP patients were triaged by physiotherapists within 2 weeks at the ORT SOPC, PWH. The triage was based on the standard spinal assessment forms and the absent of "red flags" indicative of possible serious spinal pathology such as cauda equina, inflammatory arthritis, malignancy, infection and fracture. After that, patients were classified to urgent, semi-urgent and routine (non-urgent) cases and brief self-management education was given. All the triaged reports would be checked again by an ORT specialist to confirm the priority. The waiting time at the ORT SOPC is 1 week for urgent cases, 5 weeks for semi-urgent cases and 182 weeks for routine cases. All patients with routine appointment and fulfilled the eligibility criteria were invited to participate in the study. Inclusion criteria were participants aged $\geq 18$ years old; low back pain of any episodic duration; with or without referred leg pain; and understand Chinese (Cantonese/Mandarin) to a level where they can read the questionnaires and consent forms. Exclusion criteria were participants in active work compensation claims; pregnancy related LBP; and co-morbidity severe enough to prevent participation in the model, like attendance at scheduled appointments.

The PRIOR LBP care model: 
Eligible participants were referred to primary care physicians with knowledge in musculoskeletal medicine at the FM-ORT clinic for clinical assessment and management. The roles of our primary care physicians were to confirm the diagnosis of LBP and to pick up subtle signs or symptoms suggestive of higher prognostic risks, which would need early interventions or orthopaedics care. The attending physicians would also conduct biomechanical assessment, posture evaluation and muscle testing. Postural advice, simple manual therapy included muscle energy technique and trigger points' release, and focused exercise prescriptions were offered. Appropriate patients were referred to receive physiotherapy and/or occupational therapy; medications included the use of paracetamols and non-steroidal inflammatory drugs.

\section{Baseline data collection:}

The data included age, sex, body mass index (BMI), occupation, duration of LBP and types of previous treatment modalities. Participants were asked to fill in the Subgroup for Targeted Treatment (STarT) screening tool. ${ }^{15}$ It is a simple prognostic questionnaire that helps clinicians to identify modifiable risk factors (biomedical, psychological and social) for back pain disability. The tool consists of 9 self-reported items capturing 8 prognostics factors: function ( 2 items), radiating leg pain, pain elsewhere, depression, anxiety, fear avoidance, catastrophizing, and bothersomesness. The score ranges from 0 to 9 and allocates patients into 3 risk groups: low, medium, or high risk of persistent disability. ${ }^{16}$

Outcome measurements at 3 and 6 months:

\section{Patient-specific outcomes:}

Patient acceptance to the model was measured by the recruitment rate. The Ronald Morris Disability Questionnaire (RDQ) is a self-report health status measure to assess physical disability due to low back pain. ${ }^{17}$ The RDQ score is calculated by adding up the number of items checked. The score ranges from 0 (no disability) to 24 (maximum disability). A validated Hong Kong Chinese version of RMDQ will be used in this study. ${ }^{18}$ The Chinese Pain Self-Efficacy questionnaire, with scores rated from 0-60, is an important psychosocial determinant of pain behavior and predict the outcome of pain management programs with good psychometric properties. ${ }^{19-21}$ The overall pain was measured by the Numeric Pain Rating Scale from 0 to 10. The numerical global rating of change of scale was also measured, with -10 for the worse change, and +10 for the best change. The health-related quality of life was measured by Euro-Qol-5D. ${ }^{22}$ An open question on "How do you find your care under this model?" was asked at the 6 months assessment. The numbers of adverse events from treatment were also measured.

\section{Health service outcomes:}

Health service outcomes included the average waiting time for the first FM-ORT consultation, the average number of FM-ORT consultations, the proportion of patients successfully discharged from clinic (discharge rate), the dropout rate from the care model, and the proportion of patients being referred back to ORT SOPC at 6 months. Resources utilization included the number of referrals to physiotherapy and occupational therapy, the number of patients prescribed analgesic medications and the number of patients arranged for Magnetic Resonance Imaging (MRI). 


\section{Statistical analysis:}

Sample size calculation:

A sample size of 30 had $48 \%$ power to detect an acceptable rate of $85 \%$ or lower (in terms of recruitment rate), assuming the true acceptance rate in the population was $95 \%$ using one-sided binomial test of size $a=$ 0.05 .

\section{Statistical analysis:}

We used the chi-square test to assess the recruitment rates against the pre-specified thresholds. We conducted a linear mixed models (LMM) analysis for patient-specific outcomes to investigate significant changes over time following the intention to treat principle. One-sample T-test was used for NGRCS. In the LMM models, outcomes were treated as the dependent variables, with time and baseline characteristics treated as covariates. Time was tested as a categorical variable. Age, gender, BMI, back pain duration and the respective baseline outcome were entered to the models for adjustment. Unstructured covariance structure and fixed effect parameters were applied. The statistical package Stata version 24.0 was employed for data analysis. A P value $<0.05$ was considered statistically significant.

\section{Results}

The overall study work flow was summarized in Figure 1.

Participants were recruited from 19th April 2018 to 26th July 2018. Of the 33 participants screened, 30 were eligible and enrolled for the study, and the recruitment rate was $91 \%(X 2=0.569, p=0.451)$. All 30 participants completed the baseline questionnaire. At 6 months, 3 patients withdrawn from study ( 2 were lost to followup and 1 refused to answer the questionnaires).

The study sample ( $51.8 \pm 13.1$ years old, 20 women) had a BMI of $24.0 \pm 3.3 \mathrm{~kg} / \mathrm{m} 2$, and LBP duration for $3.1 \pm 2.7$ years. It consisted of $33.3 \%$ low risk, $46.7 \%$ medium risk and $16.7 \%$ high risk according to the STarT Screening Tool. (Table 1)

Participants reported overall improvement of the RMD scores ( $\beta$ coefficient $-4.3,95 \%$ confidence interval $\mathrm{Cl}$ -5.9 to $-2.7, \mathrm{P}<0.001$ ), PSEQ scores ( $\beta$ coefficient 7.3, 95\% Cl 3.0-12.0, $P=0.002$ ), NPRS ( $\beta$ coefficient -1.5 , $95 \% \mathrm{Cl}-2.5$ to $-0.15, \mathrm{P}=0.004$ ) and NGRCS (mean difference $4.3,95 \% \mathrm{Cl} 2.6-6.0, \mathrm{P}<0.001$ ) at 6 months. (Table 2 and 3) Twenty-four out of the 27 participants were satisfied with the service model; they reported "earlier appointment", "better knowledge", "much relieved worries" and "earlier recovery". No adverse events were reported in this study.

The mean waiting time of the FM-ORT clinic was $11.6 \pm 1.6$ weeks. At 6 months, the overall discharge rate was $73 \%$, among which $33.3 \%$ could be discharged after a single consultation. The mean number of consultations before discharge was $2.13 \pm 1.0$. The adherence rate to the service model was $87 \%$. Two participants belonging to the STarT high risk groups were eventually referred back to the ORT SOPC for further management. A total of 22 participants were referred to receive a course of physiotherapy, each 
attended $4.9 \pm 5.3$ treatment sessions; 3 participants were referred to occupational therapy, each attended $4.4 \pm 2.0$ treatment sessions. Only 4 participants were prescribed analgesic medications during the visit; none of participants were arranged MRI.

\section{Discussion}

Our study suggested that the PRIOR LBP care model based on the context of local healthcare system could potentially improve patient-specific and healthcare service outcomes. We identified that the key elements of success in this model were an appropriate triage service and a trained primary care physician in musculoskeletal knowledge and skills.

The STarT screening tool is developed by the Keele University in United Kingdom. The aim is to use the stratified risk approach to assist the decision-makings of primary care physicians in the LBP management. However, its clinical application has been limited in our local settings, given that referrals to ORT SOPC can be made from various specialties. Therefore, its use in the current model was applied in the triage phase at the ORT SOPC. The tool successful triaged low and medium risks patients who were able to be managed by primary care physicians. For high risks patients, $40 \%$ of them still need to refer back to orthopedic surgeons. The management of STarT high-risk patients may need further exploration. The absence of orthopedic interventions at the initial triage phase may imply that their benefit from an orthopedic consultation would be minimal. Measures such as incorporating multidisciplinary pain service and allocating more intensive psychosocial care at this juncture may be indicated.

Musculoskeletal complaints account for $10 \%$ to $15 \%$ of all visits to primary care physicians, and $70 \%$ of all new musculoskeletal injuries are treated by primary care physicians. ${ }^{23}$ Although $90 \%$ of common nonsurgical orthopedic complaints are thought to be manageable in the primary care setting, the musculoskeletal exam and procedures are often inadequately performed by primary care physicians at all levels of training ${ }^{24}$ Previous research has suggested that when primary care physicians are provided additional training in musculoskeletal conditions, their confidence in managing these conditions increased and referrals are reduced. ${ }^{25,26}$ Therefore, we propose to establish a structured musculoskeletal training curriculum with the goal of providing post-graduate training to primary care physicians. The content may include biomechanical assessment, simple manual therapy, postural advice and exercise prescriptions etc.

The current study findings were further supported by the shortened waiting time at the ORT SOPC. According to our statistics, the waiting time of ORT SOPC was reduced from 182 weeks to 143 weeks since the commencement of the FM-ORT clinic at the New Territory East Region. The shortened waiting time was encouraging, suggesting that the collaboration between primary care physicians and orthopedic surgeons could streamline the care of LBP patients, and be one of the potential solutions to reduce the overall LBP disease burden in our local health care system. Our model also supported that not all LBP patients would need to see an ORT surgeon; those without surgical interventions could be safely managed by primary care physicians and allied health professionals. 
The study has some limitations. The interpretation was restricted by the lack of comparison group, the small sample size and the failure to exclude the natural history of LBP as a potential confounder. Our study warrants further evaluation by implementing a larger scale multi-centers randomized controlled trials with comparative analysis to routine care. Besides, a cost-effectiveness study may further provide indications on resources allocation. Qualitative views from different stakeholders, such as primary care physicians, orthopedic surgeons, nurses, physiotherapists, occupational therapists, patients other administrative personnel will be essential for overall service evaluation.

\section{Conclusion}

The study suggested that the PRIOR LBP model with collaborative input from primary care physicians, orthopaedic surgeons and allied health professionals, could potentially improve the clinical outcomes of LBP patients and the overall LBP health service delivery. Future larger scale clinical trials with costeffectiveness analysis will help to confirm its overall effectiveness and provide implication on resources allocation.

\section{Declarations}

\section{Ethical Statement:}

The study was jointly approved by the Ethics Committee of the Chinese University of Hong Kong and the New East Territories East Cluster Clinical Research Ethics Committee (CREC no.: 2017.316). Written informed consent was obtained from all participants.

\section{Consent for publication}

Not applicable.

\section{Availability of data and materials}

The datasets used and analysed during the current study are available from the corresponding author on reasonable request.

\section{Competing interests}

The authors declare that they have no competing interests.

\section{Authors' contributions}

RWSS was the principal investigator of the study and responsible for the study design and operation, data interpretation and the major contributor in write up of the manuscript. SYKC was responsible for the study design, service operation and write up of the manuscript. WWLW and JL were responsible for study design and physiotherapy care. SWL was responsible for the study design and orthopedic care. SYK and SL were responsible for data collection and analysis. EH and SYSW contributed to the conception and study design, data interpretation and writing up of the manuscript. All authors read and approved the final manuscript. 
Not applicable.

\section{Abbreviations}

LBP: Low back pain; PRIOR: primary-orthopaedic; ORT: orthopaedic; SOPC: specialist outpatient clinics; PWH: Prince of Wales Hospital; FM-ORT: Family Medicine-Orthopaedic Clinic; BMI: body mass index; STarT: Subgroup for Targeted Treatment; RMDQ: Ronald Morris Disability Questionnaire; MRI: Magnetic Resonance Imaging;PSE: self-pain efficacy scores; NGRCS: Numeric Global Rating of Change Scale;LMM: linear mixed models

\section{References}

1. Hoy D, March L, Brooks P, et al. The global burden of low back pain: estimates from the Global Burden of Disease 2010 study. Annals of the rheumatic diseases. 2014;73(6):968-974.

2. Hartvigsen J, Hancock MJ, Kongsted A, et al. What low back pain is and why we need to pay attention. The Lancet. 2018.

3. James SL, Abate D, Abate KH, et al. Global, regional, and national incidence, prevalence, and years lived with disability for 354 diseases and injuries for 195 countries and territories, 1990-2017: a systematic analysis for the Global Burden of Disease Study 2017. The Lancet. 2018;392(10159):1789-1858.

4. Prince MJ, Wu F, Guo Y, et al. The burden of disease in older people and implications for health policy and practice. The Lancet. 2015;385(9967):549-562.

5. Dagenais S, Caro J, Haldeman S. A systematic review of low back pain cost of illness studies in the United States and internationally. The spine journal. 2008;8(1):8-20.

6. Hong J, Reed C, Novick D, Happich M. Costs associated with treatment of chronic low back pain: an analysis of the UK General Practice Research Database. Spine. 2013;38(1):75-82.

7. Maher C, Underwood M, Buchbinder R. Non-specific low back pain. The Lancet. 2017;389(10070):736747.

8. Foster NE, Anema JR, Cherkin D, et al. Prevention and treatment of low back pain: evidence, challenges, and promising directions. The Lancet. 2018.

9. Koes BW, Van Tulder M, Lin C-WC, Macedo LG, McAuley J, Maher C. An updated overview of clinical guidelines for the management of non-specific low back pain in primary care. European Spine Journal. 2010;19(12):2075-2094.

10. The Hospital Authority HKSAR. 2017; http://ha.org.hk/visitor/sopc_waiting_time.asp?id=5\&lang=ENG.

11. Lynch ME, Campbell F, Clark AJ, et al. A systematic review of the effect of waiting for treatment for chronic pain. Pain. 2008;136(1-2):97-116.

12. Croft PR, Macfarlane GJ, Papageorgiou AC, Thomas E, Silman AJ. Outcome of low back pain in general practice: a prospective study. Bmj. 1998;316(7141):1356. 
13. Staal J, Hlobil $\mathrm{H}$, Van Tulder $\mathrm{M}$, et al. Occupational health guidelines for the management of low back pain: an international comparison. Occupational and Environmental Medicine. 2003;60(9):618-626.

14. Lynch ME, Campbell F, Clark AJ, et al. A systematic review of the effect of waiting for treatment for chronic pain. PAIN. 2008;136(1):97-116.

15. Hill JC, Whitehurst DG, Lewis M, et al. Comparison of stratified primary care management for low back pain with current best practice (STarT Back): a randomised controlled trial. The Lancet. 2011;378(9802):1560-1571.

16. Sowden G, Hill JC, Konstantinou K, et al. Targeted treatment in primary care for low back pain: the treatment system and clinical training programmes used in the IMPaCT Back study (ISRCTN 55174281). Family Practice. 2012;29(1):50-62.

17. Roland M, Morris R. A study of the natural history of back pain: part I: development of a reliable and sensitive measure of disability in low-back pain. Spine. 1983;8(2):141-144.

18. Tsang RC. Measurement properties of the Hong Kong Chinese version of the Roland-Morris disability questionnaire. Hong Kong Physiotherapy Journal. 2004;22(1):40-49.

19. Nicholas MK. The pain self-efficacy questionnaire: Taking pain into account. European journal of pain (London, England). 2007;11(2):153-163.

20. Maughan EF, Lewis JS. Outcome measures in chronic low back pain. European Spine Journal. 2010;19(9):1484-1494.

21. Lim HS, Chen PP, Wong TC, et al. Validation of the Chinese version of pain self-efficacy questionnaire. Anesth Analg. 2007;104(4):918-923.

22. Herdman $M$, Gudex $C$, Lloyd A, et al. Development and preliminary testing of the new five-level version of EQ-5D (EQ-5D-5L). Quality of life research. 2011;20(10):1727-1736.

23. Houston TK, Connors RL, Cutler N, Nidiry MA. A primary care musculoskeletal clinic for residents: success and sustainability. J Gen Intern Med. 2004;19(5 Pt 2):524-529.

24. Matzkin E, Smith MEL, Freccero CD, Richardson AB. Adequacy of education in musculoskeletal medicine. JBJS. 2005;87(2):310-314.

25. Åkesson K, Dreinhöfer KE, Woolf AD. Improved education in musculoskeletal conditions is necessary for all doctors. Bulletin of the World Health Organization. 2003;81:677-683.

26. Roberts $C$, Adebajo A, Long S. Improving the quality of care of musculoskeletal conditions in primary care. Rheumatology. 2002;41(5):503-508.

\section{Tables}

Table 1. Baseline characteristics of participants, mean \pm SD/ count (col\%) 


\begin{tabular}{ll}
\hline & Total $(\mathrm{N}=30)$ \\
\hline Age (years) & $51.8 \pm 13.1$ \\
BMI (kg/m2) & $24.0 \pm 3.3$ \\
Gender & \\
Male & $10(33.3 \%)$ \\
Female & $20(66.7 \%)$ \\
Occupation & \\
Retired & $6(20 \%)$ \\
Housewife & $6(20 \%)$ \\
Employed & $18(60 \%)$ \\
Back pain duration (years) & $3.1 \pm 2.7$ \\
Previous treatment & \\
Acupuncture & $7(23.3 \%)$ \\
Traditional Chinese Medicine & $10(33.3 \%)$ \\
Physiotherapy & $9(30 \%)$ \\
Tui Na & $3(10 \%)$ \\
Bone Setting & $2(6.7 \%)$ \\
None & $12(40 \%)$ \\
STarT Back(total) & $4.4 \pm 2.0$ \\
STarT Back(subscore) & $2.1 \pm 1.5$ \\
STarT Back(category of risk) & \\
Low risk & $10(33.3 \%)$ \\
Medium risk & $14(46.7 \%)$ \\
High risk & $5(16.7 \%)$ \\
\hline
\end{tabular}

BMI = Body Mass Index, STarT Back= The Keele STarT Back Screening Tool

Table 2. Effect of treatment on different outcome measures $(n=30)$, mean $\pm S D$, using linear mixed model 


\begin{tabular}{lccc}
\hline \multicolumn{3}{c}{ Adjusted for baseline characteristics \& baseline outcome measure } \\
\hline Mean \pm SD & $\beta$ coefficient (95\% CI) & p-value* \\
\hline Baseline & $7.1 \pm 4.1$ & & $<0.001$ \\
\hline 3 Month & $3.8 \pm 3.0$ & $-3.5(-5.2,-1.7)$ & $<0.001$
\end{tabular}

PSEQ

Baseline $\quad 39.4 \pm 12.3$

3 Month $\quad 45.9 \pm 10.4$

$6.2(2.0,10.3)$

0.005

6 Month 46.6 12.2

$7.5(3.0,12.0)$

0.002

EQ5D index

Baseline $\quad 0.6 \pm 0.3$

3 Month

$0.7 \pm 0.2$

$0.1(-0.1,0.2)$

$-0.115$

6 Month

$0.7 \pm 0.2$

$0.1(-0.1,0.2)$

0.052

\section{EQVS}

Baseline $\quad 68.6 \pm 18.4$

3 Month $\quad 68.2 \pm 14.4$

$-0.6(-8.2,7.0)$

0.872 
6 Month $\quad 70.0 \pm 16.1$

$1.1(-6.8,9.0)$

0.781

NPRS

Baseline $\quad 5.2 \pm 2.3$

3 Month

$3.9 \pm 2.2$

6 Month

$3.7 \pm 2.7$
$-1.3(-2.2,-0.4)$

$-1.5(-2.5,-0.5)$
0.005

0.004

$\mathrm{RMDQ}=$ Ronald Morris Disability questionnaire, PSEQ= Pain Self-Efficacy questionnaire, EQ5D = EuroQol-5D, EQVS = EuroQol-5D Visual Analogue Scale, NPRS $=$ Numeric Pain Rating Scale. All the models were adjusted for age, gender, BMI, back pain duration, STarT and other baseline scores.

Table 3. Mean differences in NGRCS scores using one-sample T-test

\begin{tabular}{rccc}
\hline & Mean \pm SD & Mean Difference (95\% CI) & p-value* \\
\hline NGRCS & & & \\
\hline 3 Month & $3.5 \pm 4.2$ & $3.5(1.9,5.2)$ & $<0.001$ \\
& & & \\
\hline 6 Month & $4.3 \pm 4.4$ & $4.3(2.6,6.0)$ & $<0.001$ \\
\hline
\end{tabular}

NGRCS $=$ Numeric Global Rating of Change Scale

\section{Figures}



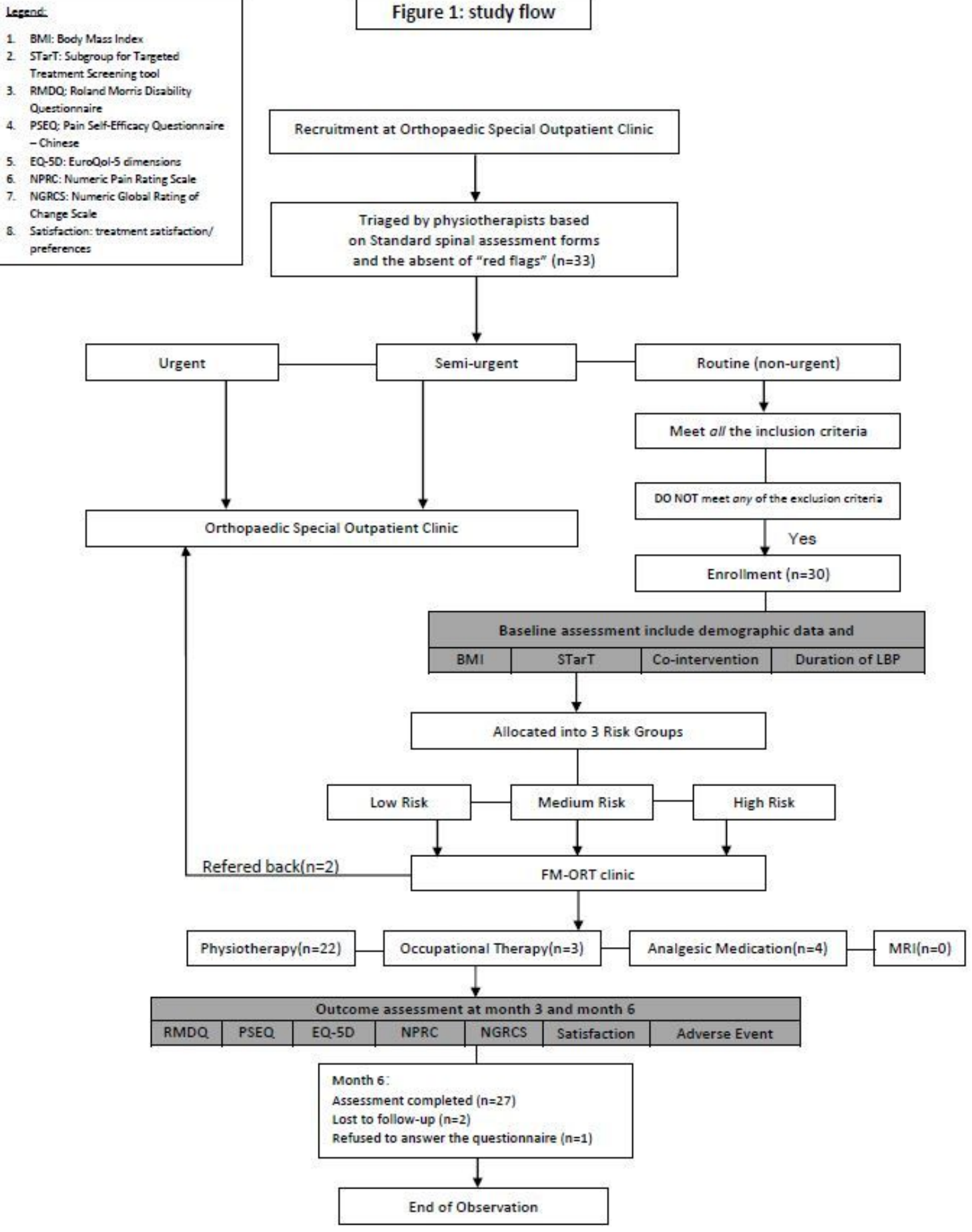

\section{Figure 1}

Study flow. 